Supporting information for

\title{
Electroplated Silver-Nickel Core-Shell Nanowire Network Electrodes for Highly Efficient Perovskite Nanoparticle Light-Emitting Diodes
}

Hyungseok Kang, ${ }^{1, \uparrow}$ Seok-Ryul Choi, ${ }^{2, \dagger}$ Young-Hoon Kim, ${ }^{3}$ Joo Sung Kim, ${ }^{3}$ Sungjin Kim,,${ }^{3}$ Byeong-Seon An, ${ }^{2}$ Cheol-Woong Yang, ${ }^{2}$ Jae-Min Myoung, ${ }^{4}$ Tae-Woo Lee, ${ }^{3}$ Jung-Gu Kim, ${ }^{2, *}$ Jeong Ho Cho ${ }^{5 * *}$

${ }^{1}$ SKKU Advanced Institute of Nanotechnology (SAINT), ${ }^{2}$ School of Advanced Materials Science and Engineering, Sungkyunkwan University, Suwon 440-746, Republic of Korea.

${ }^{3}$ Department of Materials Science and Engineering, BK21 PLUS SNU Materials Division for Educating Creative Global Leaders, Research Institute of Advanced Materials, Institute of Engineering Research, Nano System Institute (NSI), Seoul National University, Seoul 08826, Republic of Korea.

${ }^{4}$ Department of Materials Science and Engineering, ${ }^{5}$ Department of Chemical and Biomolecular Engineering, Yonsei University, Seoul 03722, Republic of Korea.

$\dagger^{\dagger}$ H. Kang and S.-R. Choi contributed equally to this work.

*Corresponding authors: J. H. Cho (jhcho94@yonsei.ac.kr) and J.-G. Kim (kimjg@skku.edu)

Keywords: perovskite nanoparticle, electroluminescence efficiency, silver nanowire, electroplating, transparent electrode 


\section{METHODS}

Ni electroplating and characterization $\mathrm{Ni}$ was electrodeposited from an electrolyte containing $150 \mathrm{~g} / \mathrm{L}$ $\mathrm{NiSO}_{4}, 15 \mathrm{~g} / \mathrm{L} \mathrm{NH}{ }_{4} \mathrm{Cl}$, and $15 \mathrm{~g} / \mathrm{L} \mathrm{H}_{3} \mathrm{BO}_{3}$, with the $\mathrm{pH}$ values of the electrolyte ranging between 5.5 and 6 . A two-electrode cell $(500 \mathrm{~mL})$ was used for the electrodeposition process. The AgNWs $(0.5 \mathrm{wt} \%$, Nanopyxis Co.) with diameter of $30 \mathrm{~nm}$ and length of $30 \mu \mathrm{m}$ were deposited onto the substrate through the Meyer-rod coating (\#7). The prepared AgNW networks (working electrode) were dipped in the Ni electroplating bath. The counter electrode was a Pt mesh. The charge densities were varied $(3.8,7.5,15,19$, and $38 \mathrm{mC} / \mathrm{cm}^{2}$ ). LSV was performed with a constant electrode-rotation speed (500 rpm). The electrode potential was linearly swept from $-0.8 \mathrm{~V}$ to $-2.5 \mathrm{~V}$ versus the Pt electrode at a sweep rate of $20 \mathrm{mV} / \mathrm{s}$. The pristine and electrodeposited AgNWs were visualized using SEM (JEOL JSM-7600F) and TEM (JEOL JEM ARM200F). The sheet resistance and optical transmittance were measured with a four-point probe method (Keithley 2182A nanovoltmeter and Keithley 6221 current source) and a UV-vis spectrophotometer (Agilent 8453), respectively. EIS was performed using a three-electrode system in $3.5 \mathrm{wt} \% \mathrm{NaCl}$ solution at $25^{\circ} \mathrm{C}$, by means of a VSP-300 potentiostat (BioLogic Co.). A saturated calomel electrode (SCE) and two glassy graphite rods were used as the reference electrode and counter electrode, respectively. The EIS analysis was performed at an amplitude of $\pm 10 \mathrm{mV}$ at frequencies ranging from $100 \mathrm{kHz}$ to $100 \mathrm{mHz}$.

Synthesis of $\mathrm{FAPbBr}_{3} \mathrm{NPs} 0.1 \mathrm{mmol}$ of $\mathrm{FABr}, 0.2 \mathrm{mmol}$ of $\mathrm{PbBr}_{2}$ were dissolved in $0.5 \mathrm{~mL}$ of dimethylformamide (DMF) which formed a transparent precursor solution. Next, $0.15 \mathrm{~mL}$ of precursor solution dropped into $5 \mathrm{~mL}$ of toluene solution with pre-dissolved $2 \mathrm{~mL}$ of 1-butanol, $0.3 \mathrm{~mL}$ of oleic acid and $20 \mu \mathrm{L}$ of $n$-decylamine. Direct after mixing, solution changed to yellowish color due to recrystallization of precursor. After $10 \mathrm{~min}$ mixing, the solution was centrifuged at $8000 \mathrm{rpm}$ for $10 \mathrm{~min}$. The precipitates were collected in $1 \mathrm{~mL}$ of toluene followed by centrifuge at $3500 \mathrm{rpm}$ for $10 \mathrm{~min}$ to remove large particles. Fabrication and characterization of perovskite NP LEDs The Buf-HIL composed of poly(3,4ethylenedioxythiophene):polystyrene sulfonate (PEDOT:PSS) and perfluorinated ionomer (PFI) with 1:1 $\mathrm{wt} \%$ was spin-coated onto AgNWs or Ag-Ni core-shell NWs to form a $40 \mathrm{~nm}$-thick layer and then baked at $150{ }^{\circ} \mathrm{C}$ for $30 \mathrm{~min}$. Samples were transferred to a glove box, following which perovskite NP solutions were spin-coated at $1000 \mathrm{rpm}$ for $30 \mathrm{~s}$. Subsequently, TPBI $(50 \mathrm{~nm}), \operatorname{LiF}(1 \mathrm{~nm})$, and Al $(100 \mathrm{~nm})$ were sequentially deposited in a high-vacuum chamber $\left(<10^{-7}\right.$ Torr $)$. The emitting pixel size is $6 \mathrm{~mm}^{2}$. The electrical and optical characteristics of the PeLEDs were measured with a Keithley 2400 source meter and a Minolta CS-2000 spectroradiometer. 


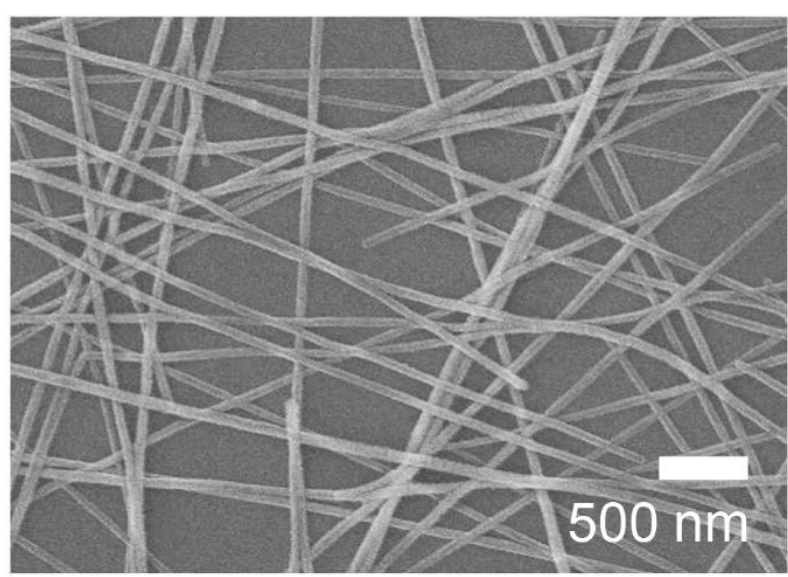

Figure S1. SEM image of the AgNWs deposited onto a PET substrate.

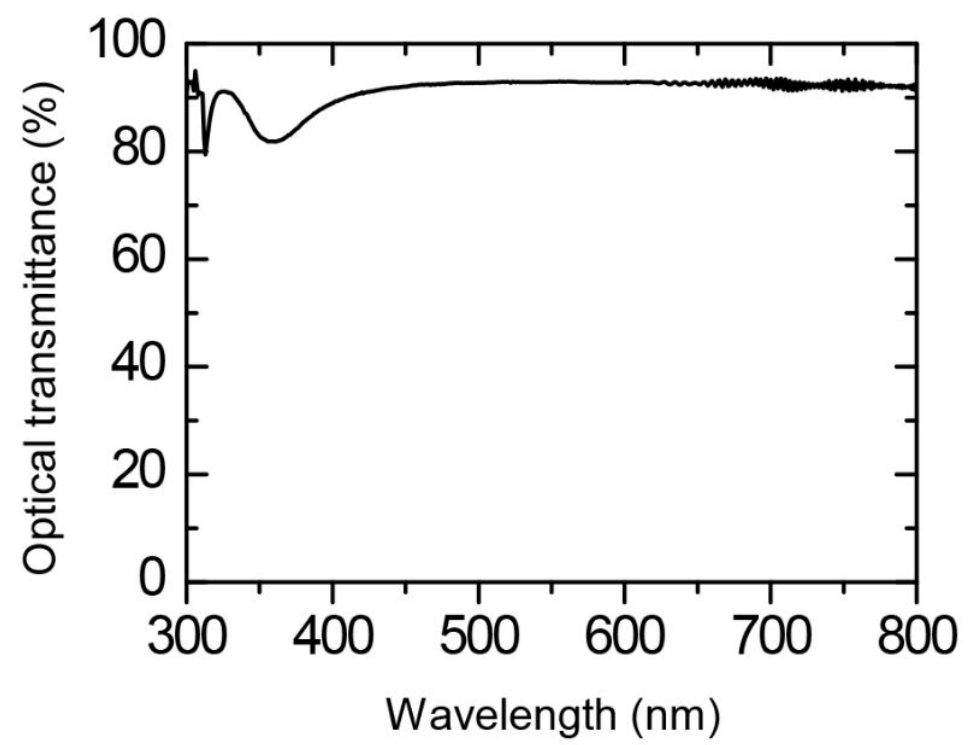

Figure S2. Optical transmittance of AgNWs deposited onto a PET film as a function of wavelength. 


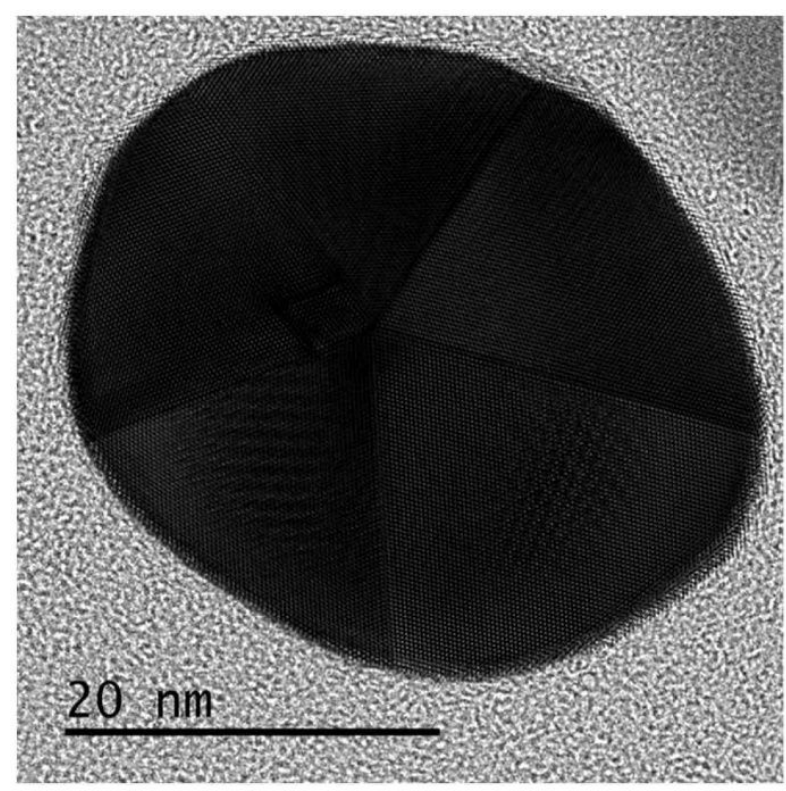

Figure S3. Cross-sectional HR-TEM image of the AgNWs in step 2. Ni was not deposited onto the AgNW surface.

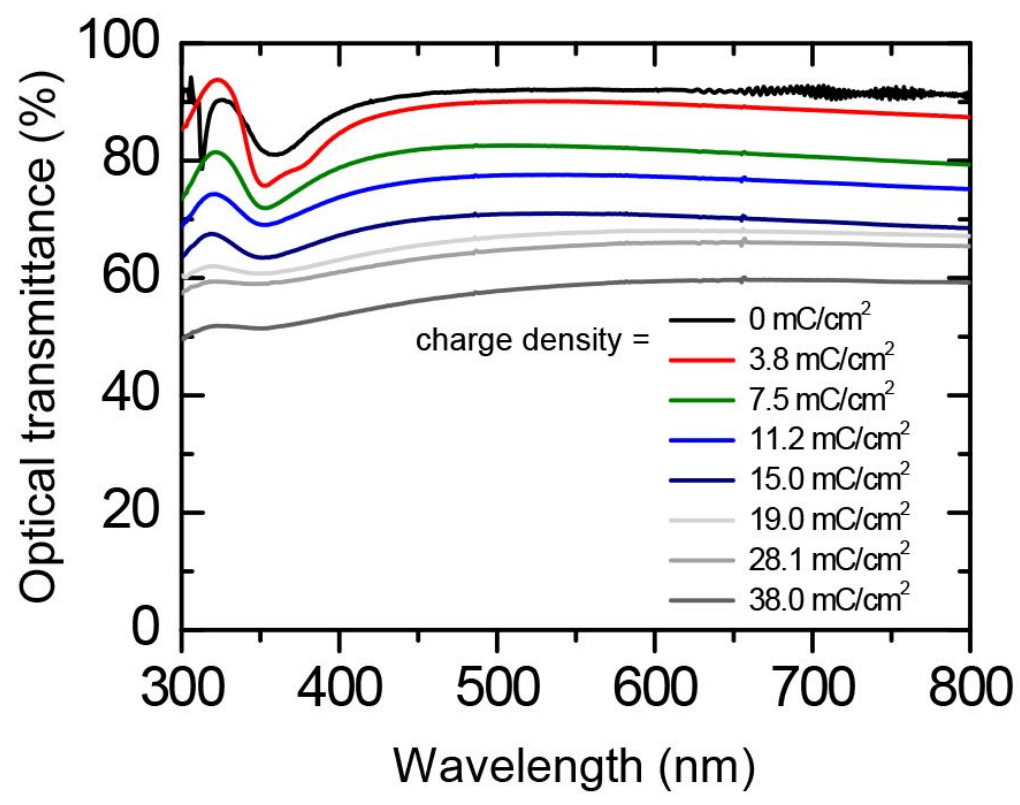

Figure S4. Optical transmittance of Ag-Ni core-shell NW film at various charge densities. 


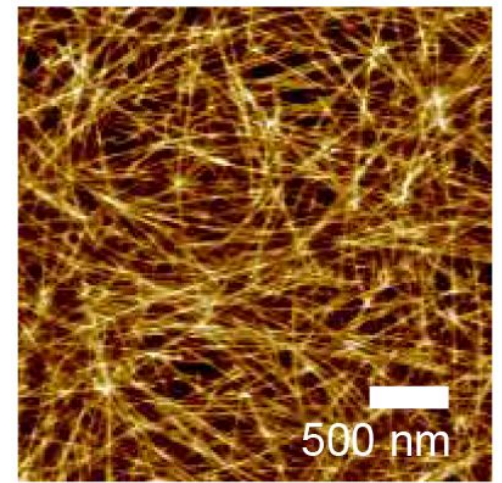

Figure S5. AFM image of the Ag-Ni core-shell NWs at charge density of $3.7 \mathrm{mC} / \mathrm{cm}^{2}$.

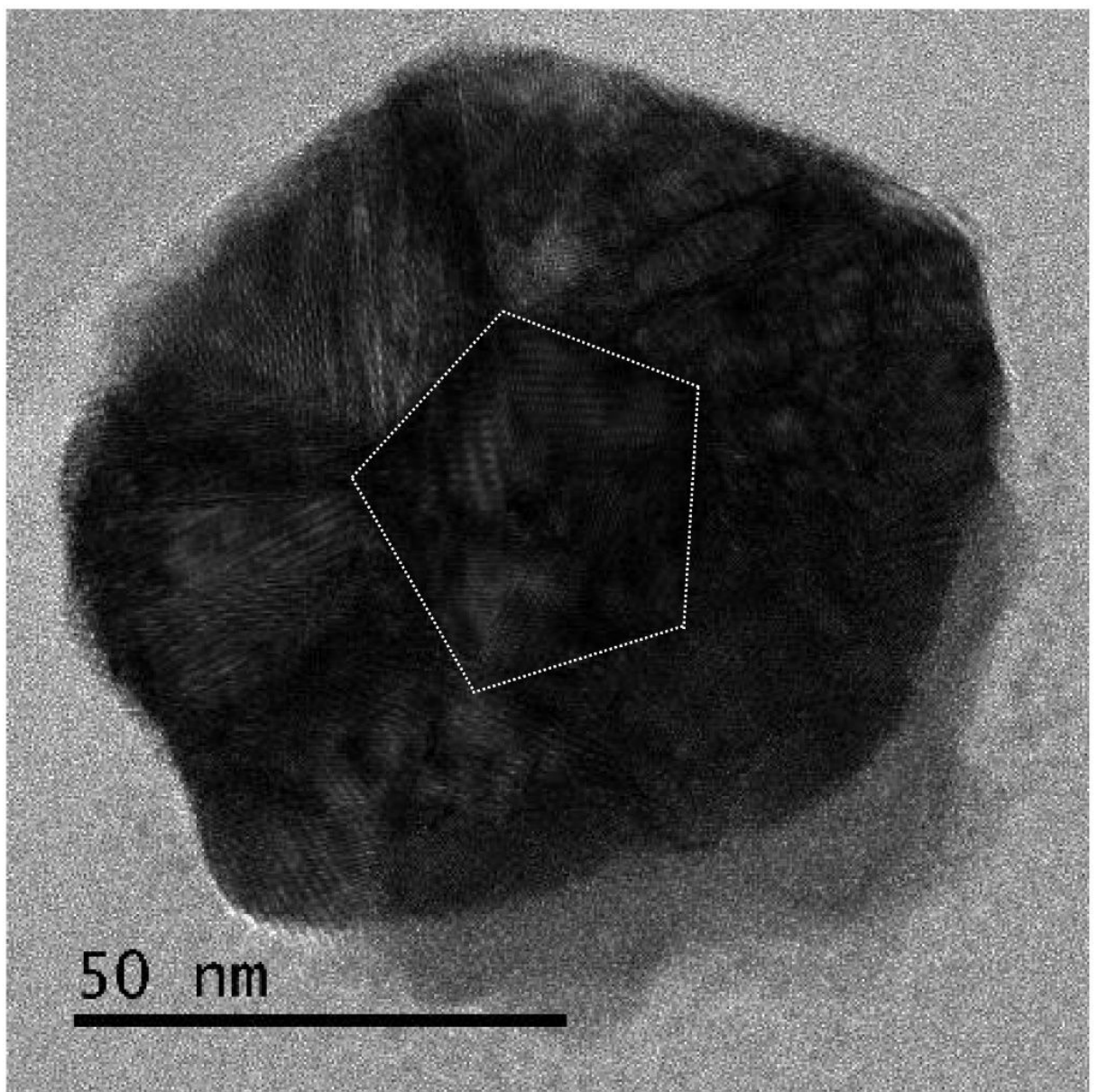

Figure S6. Cross-sectional HR-TEM image of Ag-Ni core-shell NW with thick Ni layer. 


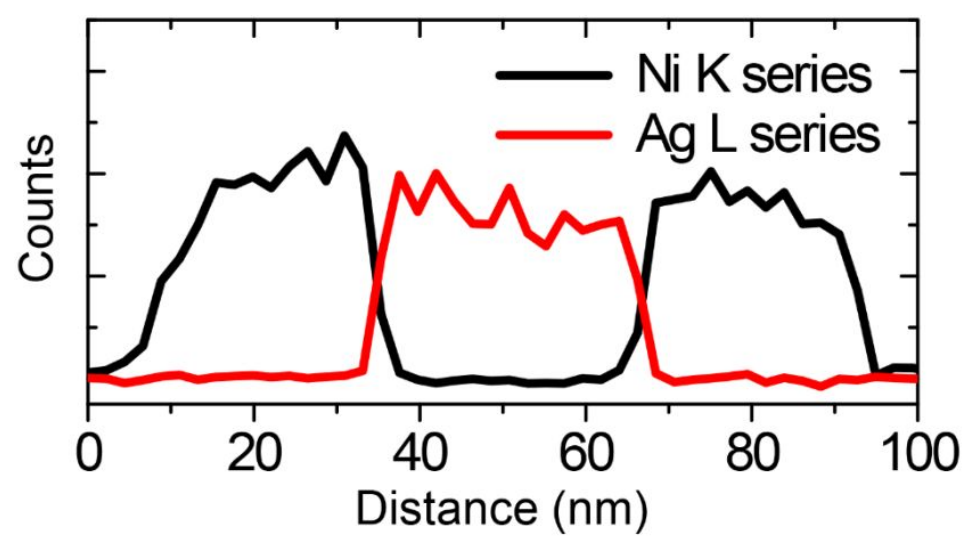

Figure S7. EDS-line-analysis results of Ag-Ni core-shell NWs.

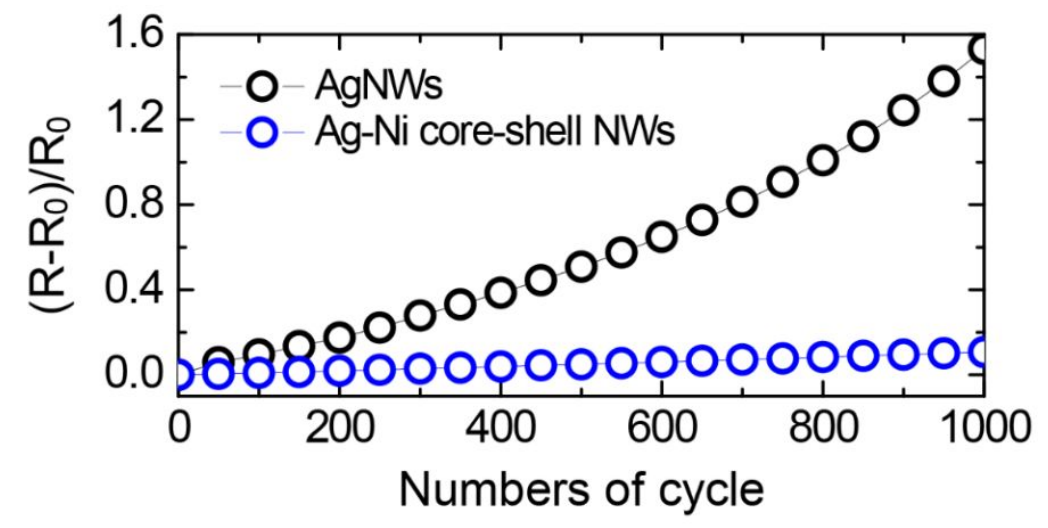

Figure S8. $\left(\mathrm{R}-\mathrm{R}_{0}\right) / \mathrm{R}_{0}$ of the metallic NWs as a function of the numbers of fatigue cycle (tensile strain $\left.=1.0 \%\right)$. $\mathrm{R}$ is the sheet resistance and $\mathrm{R}_{0}$ is the initial sheet resistance. 


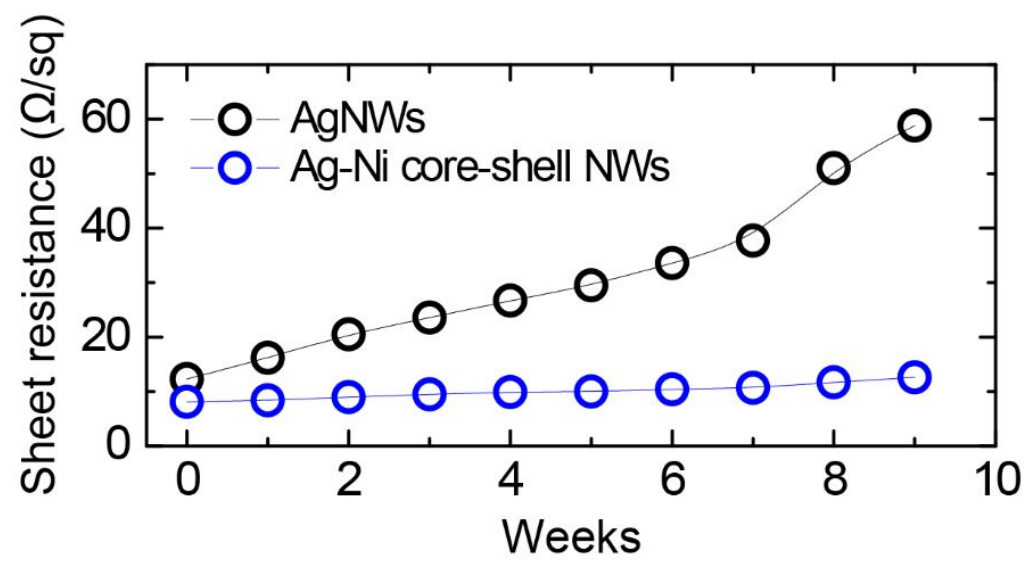

Figure S9. Environmental stability of pristine AgNWs and Ag-Ni core-shell NWs under an ambient air temperature of $22^{\circ} \mathrm{C}$ and $\mathrm{RH}$ of $\sim 73 \%$.

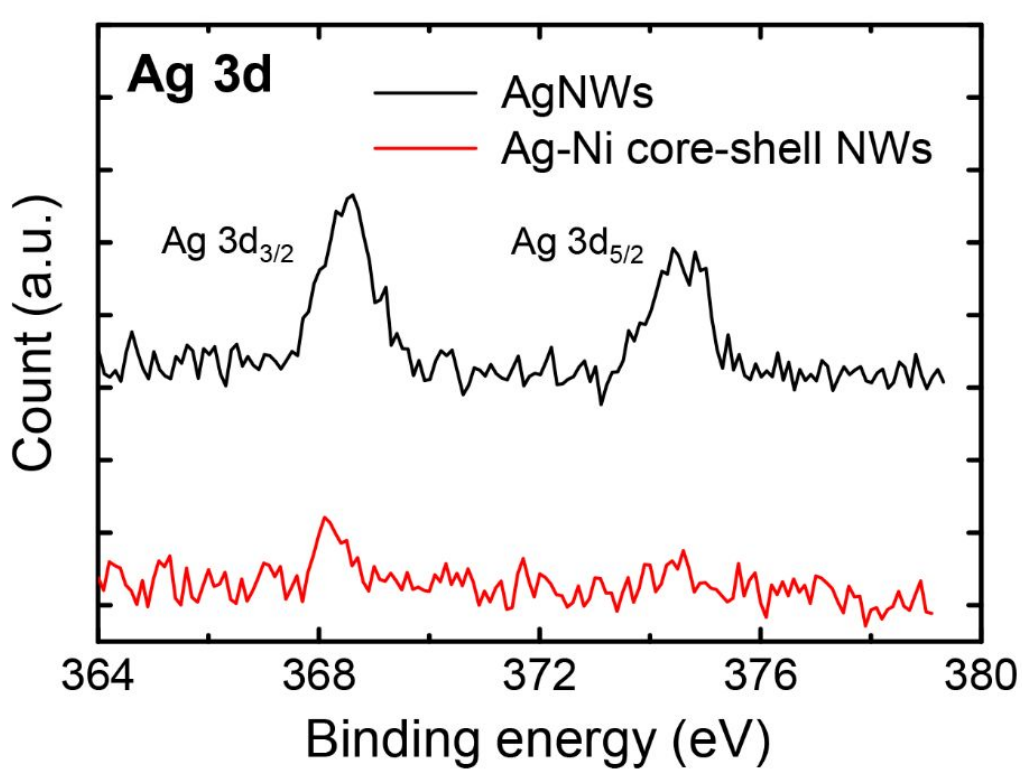

Figure S10. XPS Ag 3d spectra of the $\mathrm{FAPbBr}_{3} \mathrm{NP}$ films on AgNWs or Ag-Ni core-shell NW/Buf-HIL structures. The Ni shell suppressed the diffusion of metallic Ag ions to the perovskite NPs films. 


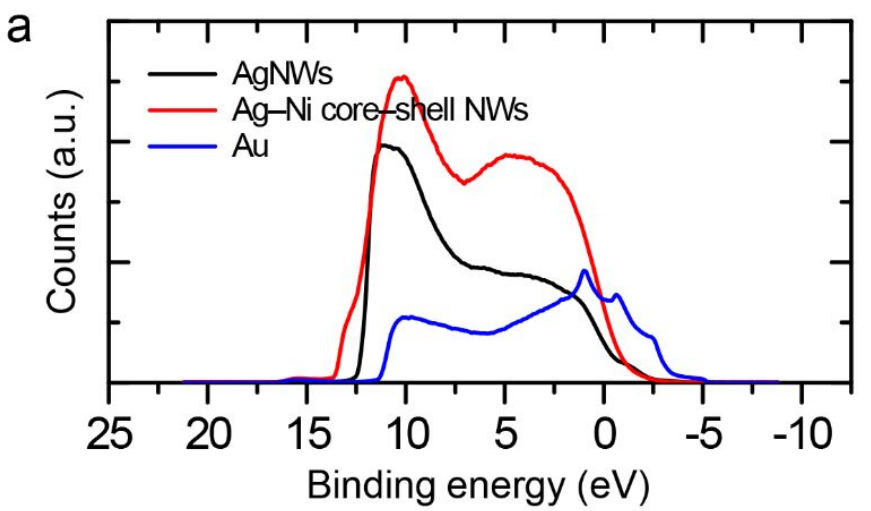

b

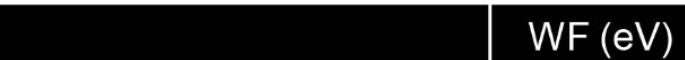

$\mathrm{Au}$

5.1

AgNWs

4.2

$\mathrm{Ag}-\mathrm{Ni}$ core-shell NWs

5.0

Figure S11. (a) UPS spectra of AgNWs, Ag-Ni core-shell NWs, and reference Au. (b) Work functions of electrodes.
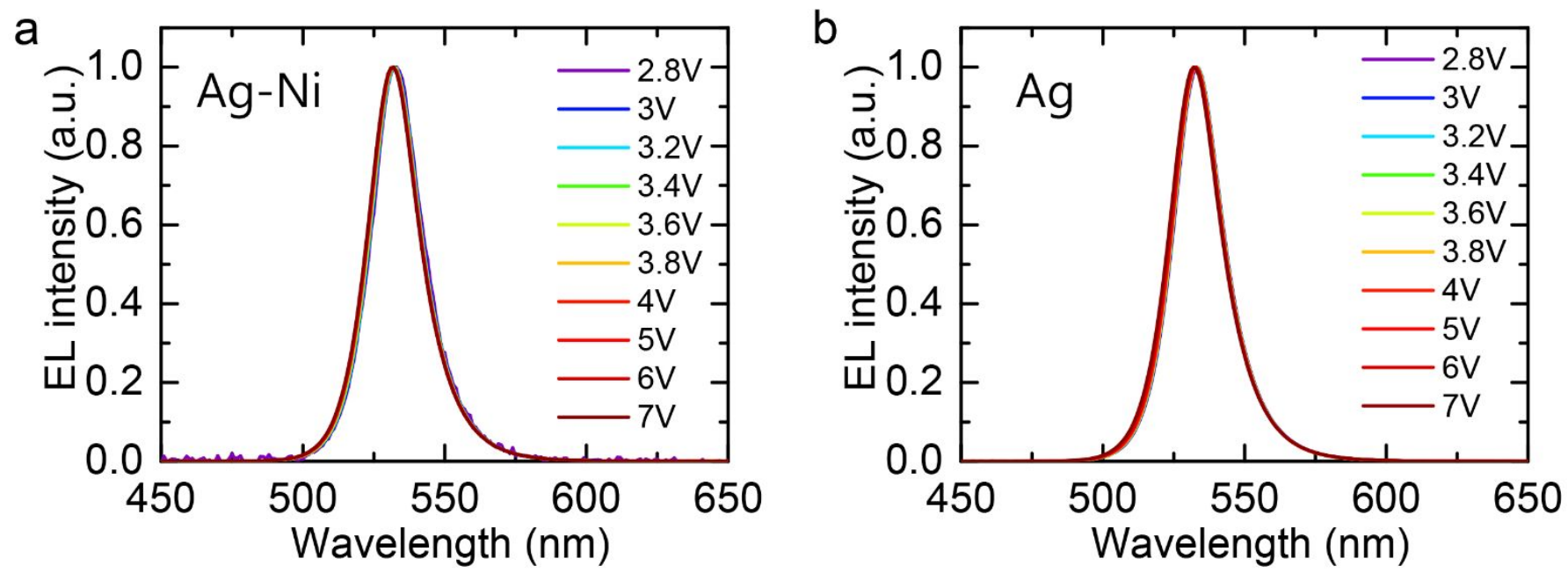

Figure S12. Electroluminescence spectra of PeLEDs with (a) Ag-Ni core-shell NWs and (b) pristine AgNWs. 